Linköping Studies in Science and Technology

Dissertations, No. 1801

\title{
TAUT STRINGS AND REAL INTERPOLATION
}

\section{Eric Setterqvist}

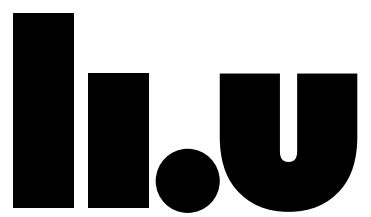

LINKÖPING UNIVERSITY

Department of Mathematics, Division of Mathematics and Applied Mathematics

Linköping University, SE-581 83 Linköping, Sweden

Linköping 2016 
Taut Strings and Real Interpolation

Eric Setterqvist

Linköping Studies in Science and Technology. Dissertations, No. 1801 ISSN 0345-7524

ISBN 978-91-7685-649-9

Copyright (c) 2016 Eric Setterqvist unless otherwise noted

Printed by LiU-Tryck, Linköping, Sweden 2016 


\section{Abstract}

The taut string problem concerns finding the function with the shortest graph length, i.e. the taut string, in a certain set $\Omega$ of continuous piecewise linear functions. It has appeared in a broad range of applications including statistics, image processing and economics. As it turns out, the taut string has besides minimal graph length also minimal energy and minimal total variation among the functions in $\Omega$.

The theory of real interpolation is based on Peetre's $K$-functional. In terms of the $K$-functional, we introduce invariant $K$-minimal sets and show a close connection between taut strings and invariant $K$-minimal sets.

This insight leads to new problems of interpolation theory, gives possibility to generalize the notion of taut strings and provides new applications.

The thesis consists of four papers. In paper I, connections between invariant $K$-minimal sets and various forms of taut strings are investigated. It is shown that the set $\Omega^{\prime}$ of the derivatives of the functions in $\Omega$ can be interpreted as an invariant $K$-minimal set for the Banach couple $\left(\ell^{1}, \ell^{\infty}\right)$ on $\mathbb{R}^{n}$. In particular, the derivative of the taut string has minimal $K$-functional in $\Omega^{\prime}$. A characterization of all bounded, closed and convex sets in $\mathbb{R}^{n}$ that are invariant $K$-minimal for $\left(\ell^{1}, \ell^{\infty}\right)$ is established.

Paper II presents examples of invariant $K$-minimal sets in $\mathbb{R}^{n}$ for $\left(\ell^{1}, \ell^{\infty}\right)$. A convergent algorithm for computing the element with minimal $K$-functional in such sets is given. In the infinite-dimensional setting, a sufficient condition for a set to be invariant $K$-minimal with respect to the Banach couple $\left(L^{1}\left([0,1]^{m}\right), L^{\infty}\left([0,1]^{m}\right)\right)$ is established. With this condition at hand, different examples of invariant $K$-minimal sets for this couple are constructed.

Paper III considers an application of taut strings to buffered real-time communication systems. The optimal buffer management strategy, with respect to minimization of a class of convex distortion functions, is characterized in terms of a taut string. Further, an algorithm for computing the optimal buffer management strategy is provided.

In paper IV, infinite-dimensional taut strings are investigated in connection with the Wiener process. It is shown that the average energy per unit of time of the taut string in the long run converges, if it is constrained to stay within the distance $r>0$ from the trajectory of a Wiener process, to a constant $\mathcal{C}^{2} / r^{2}$ where $\mathcal{C} \in(0, \infty)$. While the exact value of $\mathcal{C}$ is unknown, the numerical estimate $\mathcal{C} \approx 0.63$ is obtained through simulations on a super computer. These simulations are based on a certain algorithm for constructing finite-dimensional taut strings. 


\section{Populärvetenskaplig sammanfattning}

Denna avhandling undersöker samband mellan så kallade spända trådar och reell interpolationsteori. Med den spända trådens problem avses att finna funktionen med minimal båglängd i en särskild mängd $\Omega$ av kontinuerliga styckvis linjära funktioner. Detta problem uppkommer i olika tillämpningar inom exempelvis statistik, bildbehandling och nationalekonomi. Det visar sig att förutom minimal båglängd så har den spända tråden även minimal energi och minimal total variation i $\Omega$.

Ett viktigt verktyg i avhandlingen är $K$-funktionalen från den reella interpolationsteorin. I avhandlingen introduceras invarianta $K$-minimala mängder, givet ett Banachpar $\left(X_{0}, X_{1}\right)$, vilka innehåller ett element med minimal $K$-funktional. Invarianta $K$-minimala mängder för Banachparet $\left(\ell^{1}, \ell^{\infty}\right)$ och den spända tråden är nära sammanbundna med varandra då det visar sig att den spända trådens derivata har minimal $K$-funktional i mängden av alla derivator av funktionerna i $\Omega$.

Avhandlingen består av fyra artiklar. I artikel I utreds sambandet mellan invarianta $K$-minimala mängder för $\left(\ell^{1}, \ell^{\infty}\right)$ och spända trådar. En karakterisering ges av alla begränsade, konvexa och slutna mängder i $\mathbb{R}^{n}$ som är invarianta $K$-minimala för $\left(\ell^{1}, \ell^{\infty}\right)$.

Artikel II ger konkreta exempel på invarianta $K$-minimala mängder i $\mathbb{R}^{n}$ för $\left(\ell^{1}, \ell^{\infty}\right)$ och även en konvergent algoritm för att beräkna elementet med minimal $K$ funktional. Vidare så undersöks invarianta $K$-minimala mängder för Banachparet $\left(L^{1}\left([0,1]^{m}\right), L^{\infty}\left([0,1]^{m}\right)\right)$.

Artiklarna III och IV behandlar tillämpningar av spända trådar och invarianta $K$-minimala mängder i kommunikationsteorin respektive teorin för Wienerprocessen. 


\section{Acknowledgments}

First of all I would like to express my sincere gratitude to my supervisor Natan Kruglyak. Meeting Natan during my education in Luleå had a profound impact on my interest of doing research in mathematics. Since then, Natan has facilitated my studies in many different ways. I will single out two things which I especially treasure. First, all our discussions throughout the years which have been inspiring and helpful and provided intriguing problems. Second, the strong encouragement to also work with applications which I have benefited a lot from.

I am grateful for all help and feedback on the research progress throughout my graduate studies from my co-supervisor Irina Asekritova. Further, I highly appreciate the advice and care concerning teaching, publishing and academic life in general which Irina and Natan have given me.

Robert Forchheimer showed interest in cooperation from the very beginning of my graduate studies and introduced me to interesting problems in communication theory. Many thanks for sharing your knowledge and intellectual curiosity and for all advice.

I first met Mikhail Lifshits when he gave an interesting course on Gaussian processes. Later on cooperating with Mikhail was a rewarding experience and made a lasting impression on me. Thank you very much.

I thank everyone at the Department of Mathematics for contributing to a pleasant working atmosphere. Special thanks to the $\mathrm{PhD}$ student group for the nice activities we have done.

Thanks to George Mathai for interesting discussions concerning direction of arrival estimation and other problems in signal processing which have stimulated my interest in this area.

Finally, I thank my family and friends for their strong support and for always encouraging me to follow my interests. 


\section{List of Papers}

The thesis is based on the following papers which will be referred to in the text by their roman numerals.

I. N. Kruglyak and E. Setterqvist, Discrete taut strings and real interpolation, J. Funct. Anal. 270 (2016), 671-704.

II. N. Kruglyak and E. Setterqvist, Invariant $K$-minimal sets in the discrete and continuous settings, J. Fourier Anal. Appl., to appear.

III. R. Forchheimer and E. Setterqvist, Real-time communication systems based on taut strings, submitted.

IV. M. Lifshits and E. Setterqvist, Energy of taut strings accompanying Wiener process, Stoch. Proc. Appl. 125 (2015), 401-427.

In papers I-III, the theory and results were developed jointly by me and my coauthors. I led the writing of papers I-III. Regarding paper IV, I was actively involved in all discussions concerning the results. I constructed the algorithm for computing the energy of the discrete taut string. This algorithm I then used in simulations on a super computer for estimating the energy of the continuous taut string. I wrote the part of paper IV which concerns this work.

Parts of the papers have been presented by me at the following conferences:

1. The 10th Swedish National Computer Networking Workshop, Västerås, Sweden, June 2-3, 2014.

2. Joint Meeting of the German Mathematical Society (DMV) and the Polish Mathematical Society (PTM), Poznań, Poland, September 17-20, 2014.

3. The 11th Swedish National Computer Networking Workshop, Karlstad, Sweden, May 28-29, 2015.

4. PDEs, Potential Theory and Function Spaces, Linköping, Sweden, June 14-18, 2015.

5. Conference on Multiscale Inverse Problems, Loka Brunn, Sweden, August 2226, 2016. 


\section{Contents}

Abstract ............................... ii

Populärvetenskaplig sammanfattning . . . . . . . . . . . . . v

Acknowledgments . . . . . . . . . . . . . . . . . vii

List of Papers . . . . . . . . . . . . . . . . ix

1 Introduction 1

1.1 The taut string problem . . . . . . . . . . . . . . . . 1

1.2 Taut strings and invariant $K$-minimal sets . . . . . . . . . . . . 2

1.3 Basic notion of real interpolation ............ 5

2 Summary of papers $\quad \mathbf{9}$

2.1 Paper I: Discrete taut strings and real interpolation . . . . . . . . . . 9

2.2 Paper II: Invariant $K$-minimal sets in the discrete and continuous settings .......................... 11

2.2.1 Divergence of flows on graphs . . . . . . . . . . . . . . . . 11

2.2.2 Algorithms . . . . . . . . . . . . . . . . . . . . . 13

2.2.3 Continuous setting . . . . . . . . . . . . . . . 14

2.3 Paper III: Real-time communication systems based on taut strings . . 18

2.4 Paper IV: Energy of taut strings accompanying Wiener process . . . . 19

$\begin{array}{ll}\text { References } & 23\end{array}$

Paper I: Discrete taut strings and real interpolation 25

Paper II: Invariant $K$-minimal sets in the discrete and continuous $\begin{array}{ll}\text { settings } & 57\end{array}$

Paper III: Real-time communication systems based on taut strings 95

Paper IV: Energy of taut strings accompanying Wiener process $\quad 121$ 


\section{Introduction}

\subsection{The taut string problem}

Consider an interval $[a, b] \subset \mathbb{R}$ with $n+1, n \geq 1$, uniformly distributed points according to

$$
x_{i}=a+\frac{i(b-a)}{n}, i=0,1, \ldots, n .
$$

Let $F, G$ be two continuous piecewise linear functions on the intervals $\left[a, x_{1}\right],\left[x_{1}, x_{2}\right]$, $\ldots,\left[x_{n-1}, b\right]$ with $F \leq G, F(a)=G(a)$ and $F(b)=G(b)$. By $\Gamma_{F, G}$ we denote the set of all continuous piecewise linear functions $f$ on $[a, b]$ with nodes in $x_{i}, i=0,1, \ldots, n$, which are in between $F$ and $G$, i.e. $F \leq f \leq G$.

With the above notation introduced, we can now formulate the taut string problem as considered by G. B. Dantzig in 1971 in [8]:

Problem 1.1.1. Find the function $f_{*} \in \Gamma_{F, G}$ that satisfies

$$
\int_{a}^{b} \sqrt{1+\left(f_{*}^{\prime}(x)\right)^{2}} d x=\inf _{f \in \Gamma_{F, G}} \int_{a}^{b} \sqrt{1+\left(f^{\prime}(x)\right)^{2}} d x .
$$

It is possible to show that $f_{*}$ is unique. The function $f_{*}$ is denoted taut string as it minimizes the graph length among the functions in $\Gamma_{F, G}$. See Figure 1.1 for an illustration.

Dantzig notes in [8] that the taut string problem was discussed in 1952 in R. Bellman's seminar at RAND Corporation in connection with problems in optimal control. We would like to point out that in 1955 a special form of the taut string problem appeared in [18] in a study of certain problems in production planning. After the publication of [8], the taut string problem and its generalizations have appeared in a broad range of applications including statistics, see [1] and [15], image processing, see [23] and communication theory, see [22].

The starting point of our investigations was the following property of the taut string $f_{*}$ :

$$
\int_{a}^{b} \varphi\left(f_{*}^{\prime}(t)\right) d t=\inf _{f \in \Gamma_{F, G}} \int_{a}^{b} \varphi\left(f^{\prime}(t)\right) d t
$$

for all convex functions $\varphi: \mathbb{R} \rightarrow \mathbb{R}$. So, in particular

$$
\int_{a}^{b} \sqrt{1+\left(f_{*}^{\prime}(t)\right)^{2}} d t=\inf _{f \in \Gamma_{F, G}} \int_{a}^{b} \sqrt{1+\left(f^{\prime}(t)\right)^{2}} d t
$$




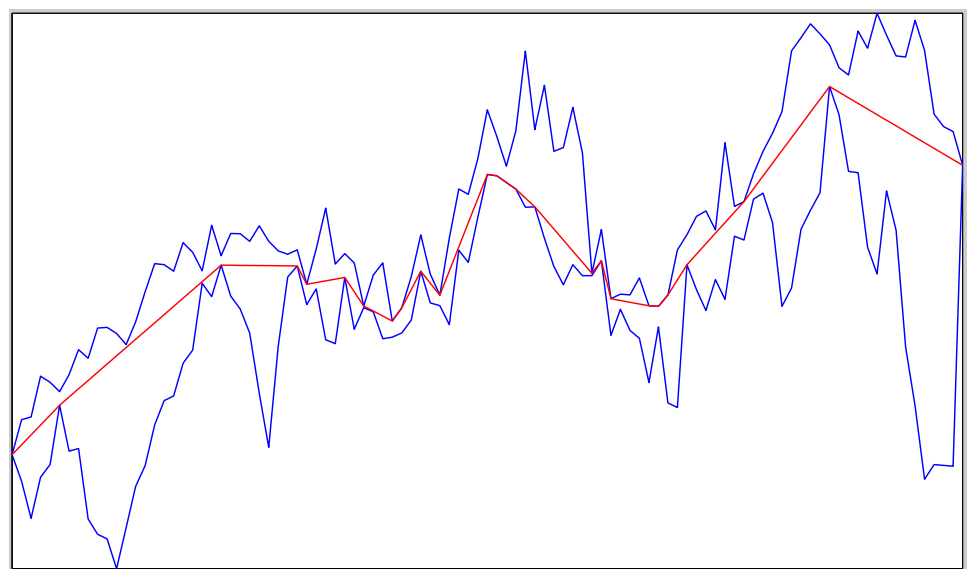

Figure 1.1: An example of a taut string (red) inside a corridor $\Gamma_{F, G}$.

$$
\int_{a}^{b}\left(f_{*}^{\prime}(t)\right)^{2} d t=\inf _{f \in \Gamma_{F, G}} \int_{a}^{b}\left(f^{\prime}(t)\right)^{2} d t
$$

and

$$
\int_{a}^{b}\left|f_{*}^{\prime}(t)\right| d t=\inf _{f \in \Gamma_{F, G}} \int_{a}^{b}\left|f^{\prime}(t)\right| d t
$$

i.e. the taut string $f_{*}$ simultaneously minimizes graph length, energy and total variation in $\Gamma_{F, G}$. Given this interesting property of the taut string and its diverse applications, our investigations was directed towards the search of other instances where similar phenomena occur.

To put this vague formulation of the research problem into a precise form, we now introduce the notion of invariant $K$-minimal sets which will provide the framework for our subsequent investigations.

\subsection{Taut strings and invariant $K$-minimal sets}

Let $\left(X_{0}, X_{1}\right)$ be a Banach couple, i.e. $X_{0}$ and $X_{1}$ are Banach spaces which are linearly and continuously embedded in some Hausdorff topological vector space $H$. The theory of real interpolation is based on Peetre's $K$-functional which for $x \in X_{0}+X_{1}$ and $t>0$ is given by

$$
K\left(t, x ; X_{0}, X_{1}\right)=\inf _{x=x_{0}+x_{1}}\left(\left\|x_{0}\right\|_{X_{0}}+t\left\|x_{1}\right\|_{X_{1}}\right) .
$$

We are now ready to give the definition of invariant $K$-minimal sets:

Definition 1.2.1 (Invariant $K$-minimal sets). Given a Banach couple $\left(X_{0}, X_{1}\right)$, a set $\Omega \subset X_{0}+X_{1}$ is called invariant $K$-minimal with respect to $\left(X_{0}, X_{1}\right)$ if for every $a \in X_{0}+X_{1}$ there exists an element $x_{*, a} \in \Omega$ such that

$$
K\left(t, x_{*, a}-a ; X_{0}, X_{1}\right) \leq K\left(t, x-a ; X_{0}, X_{1}\right)
$$


holds for all $x \in \Omega$ and every $t>0$.

Remark 1.2.1. From the definition follows that $x_{*, a}$ is the nearest element in $\Omega$ of $a \in X_{0}+X_{1}$ with respect to the norms of all exact interpolation spaces generated by the $K$-method of real interpolation. We refer to Section 1.3 for definitions of interpolation spaces and other basic notion of real interpolation.

Remark 1.2.2. From the definition follows further that if the set $\Omega \subset X_{0}+X_{1}$ is invariant $K$-minimal, then the set $\Omega+a$, given any element $a \in X_{0}+X_{1}$, is also invariant $K$-minimal. Hence, the reason for calling $\Omega$ invariant $K$-minimal.

Our work centers around the fundamental Banach couple $\left(L^{1}, L^{\infty}\right)$ where we assume that $L^{1}$ and $L^{\infty}$ are defined over a $\sigma$-finite measure space $(S, \mu)$. For this couple, the $K$-functional can be computed exactly as

$$
K\left(t, f ; L^{1}, L^{\infty}\right)=\int_{0}^{t} f^{*}(s) d s
$$

where $f^{*}$ denotes the decreasing rearrangement of $f \in L^{1}+L^{\infty}$. We recall that given a measurable function $g$ on $(S, \mu)$, the decreasing rearrangement $g^{*}$ is defined by

$$
g^{*}(t)=\inf \{\lambda: \mu(x \in S:|g(x)|>\lambda) \leq t\}
$$

From the definition follows that $g^{*}$ is nonnegative, decreasing and right-continuous on $(0, \infty)$.

In [7], A. P. Calderón established important results for the couple $\left(L^{1}, L^{\infty}\right)$. See Theorems 1.3.1, 1.3.2 and 1.3.3 in Section 1.3 for precise statements. Of special interest for us is the following consequence of Calderón's results. The inequality

$$
K\left(\cdot, g ; L^{1}, L^{\infty}\right) \leq K\left(\cdot, f ; L^{1}, L^{\infty}\right)
$$

implies that

$$
\|g\|_{L^{p}} \leq\|f\|_{L^{p}}, 1 \leq p \leq \infty .
$$

So, given an invariant $K$-minimal set $\Omega$ with respect to $\left(L^{1}, L^{\infty}\right)$ it follows that $x_{*, a}$ is the nearest element of $a$ in $\Omega$ with respect to all $L^{p}$-norms, $1 \leq p \leq \infty$.

With this result in mind, we return to the taut string problem introduced in Section 1.1 but now with the weaker assumptions $F(a) \leq G(a)$ and $F(b) \leq G(b)$. That is, $F$ and $G$ do not necessarily need to be equal at the endpoints. Such generalization is important for some applications, see Section 2.4. Define $\Omega \subset \mathbb{R}^{n}$ according to

$$
\Omega=\left\{u \in \mathbb{R}^{n}: u_{i}=\frac{f\left(x_{i}\right)-f\left(x_{i-1}\right)}{x_{i}-x_{i-1}}, i=1, \ldots, n, f \in \Gamma_{F, G}\right\} .
$$

The set $\Omega$ can be interpreted as the set of coefficient vectors of the piecewise constant derivatives of the functions in $\Gamma_{F, G}$. Take the Banach couple $\left(\ell^{1}, \ell^{\infty}\right)$ on $\mathbb{R}^{n}$ where for $x=\left(x_{1}, \ldots, x_{n}\right) \in \mathbb{R}^{n},\|x\|_{\ell^{p}}=\left(\sum_{i=1}^{n}\left|x_{i}\right|^{p}\right)^{1 / p}, 1 \leq p<\infty$, and $\|x\|_{\ell^{\infty}}=\max _{1 \leq i \leq n}\left|x_{i}\right|$. So, we consider the couple $\left(L^{1}, L^{\infty}\right)$ over the space $\{1, \ldots, n\}$ with counting measure. The following result, established in Paper I, shows the close connection between taut strings and invariant $K$-minimal sets: 
Theorem 1.2.1. The set $\Omega$ is an invariant $K$-minimal set with respect to $\left(\ell^{1}, \ell^{\infty}\right)$.

From Theorem 1.2.1 follows that the element $u_{*} \in \Omega$ with

$$
u_{*, i}=\frac{f_{*}\left(x_{i}\right)-f_{*}\left(x_{i-1}\right)}{x_{i}-x_{i-1}}, i=1, \ldots, n,
$$

i.e. the vector in $\Omega$ corresponding to the piecewise constant derivative of the taut string $f_{*} \in \Gamma_{F, G}$, has minimal $K$-functional in $\Omega$ with respect to the couple $\left(\ell^{1}, \ell^{\infty}\right)$. That is,

$$
K\left(\cdot, u_{*} ; \ell^{1}, \ell^{\infty}\right) \leq K\left(\cdot, u ; \ell^{1}, \ell^{\infty}\right), \forall u \in \Omega,
$$

which in turn implies that

$$
\left\|u_{*}\right\|_{\ell^{p}} \leq\|u\|_{\ell^{p}}, 1 \leq p \leq \infty, \forall u \in \Omega
$$

So, $u_{*}$ has minimal $\ell^{p}$-norm, $1 \leq p \leq \infty$, in $\Omega$. From (1.1), it also follows that

$$
\sum_{i=1}^{n} \varphi\left(u_{*, i}\right)=\inf _{u \in \Omega} \sum_{i=1}^{n} \varphi\left(u_{i}\right)
$$

for all even and convex functions $\varphi: \mathbb{R} \rightarrow \mathbb{R}$, see Proposition 2.1.1 in Section 2.1.

Given the close connection between taut strings and invariant $K$-minimal sets revealed by Theorem 1.2.1, some natural problems appear:

Problem 1.2.1. How to characterize the sets in $\mathbb{R}^{n}$ that are invariant $K$-minimal with respect to $\left(\ell^{1}, \ell^{\infty}\right)$ ?

Problem 1.2.2. How to efficiently compute the minimizer $x_{*, a}$ in these sets?

Problem 1.2.3. Are there new applications of taut strings and invariant $K$-minimal sets?

Problem 1.2.1 is addressed in Paper I while Problem 1.2.2 is addressed in Paper II. Investigations related to Problem 1.2.3 is partly done in Paper II and is the main focus of Papers III and IV.

Starting with the finite-dimensional setting, the next step is to search for infinitedimensional analogues which leads to the following problem:

Problem 1.2.4. How to construct invariant $K$-minimal sets with respect to the couple $\left(L^{1}\left([0,1]^{m}\right), L^{\infty}\left([0,1]^{m}\right)\right)$ ?

Paper II address this problem. 


\subsection{Basic notion of real interpolation}

The origins of interpolation theory go back to the classical Riesz-Thorin interpolation theorem [21] [24] and Marcinkiewicz interpolation theorem [16]. Introductions to interpolation theory may be found in [3], [12], [2] or [4]. Here we recall some basic notion and results.

Two Banach spaces $X_{0}$ and $X_{1}$ form a Banach couple $\left(X_{0}, X_{1}\right)$ if there is a Hausdorff topological vector space $H$ in which both $X_{0}$ and $X_{1}$ are linearly and continuously embedded. If $\left(X_{0}, X_{1}\right)$ is a Banach couple, the sum $X_{0}+X_{1}$ given by

$$
X_{0}+X_{1}=\left\{x \in H: x=x_{0}+x_{1}, x_{i} \in X_{i}, i=0,1\right\}
$$

is well-defined. One can show that $X_{0}+X_{1}$ with norm $\|\cdot\|_{X_{0}+X_{1}}$ given by

$$
\|x\|_{X_{0}+X_{1}}=\inf _{x=x_{0}+x_{1}}\left(\left\|x_{0}\right\|_{X_{0}}+\left\|x_{1}\right\|_{X_{1}}\right)
$$

is a Banach space. Further, it can be shown that the intersection $X_{0} \cap X_{1}$ of the sets $X_{0}$ and $X_{1}$ with norm $\|\cdot\|_{X_{0} \cap X_{1}}$ given by

$$
\|x\|_{X_{0} \cap X_{1}}=\max \left\{\|x\|_{X_{0}},\|x\|_{X_{1}}\right\}
$$

is a Banach space.

Let $\left(X_{0}, X_{1}\right)$ and $\left(Y_{0}, Y_{1}\right)$ be two Banach couples. A linear operator $T: X_{0}+$ $X_{1} \rightarrow Y_{0}+Y_{1}$ is referred to as a bounded linear operator from $\left(X_{0}, X_{1}\right)$ to $\left(Y_{0}, Y_{1}\right)$ if the restriction of $T$ to $X_{i}$ is a bounded operator from $X_{i}$ to $Y_{i}, i=0,1$.

Given a Banach couple $\left(X_{0}, X_{1}\right)$, a Banach space $X$ is called an intermediate space of $\left(X_{0}, X_{1}\right)$ if $X$ is linearly and continuously embedded between $X_{0} \cap X_{1}$ and $X_{0}+X_{1}$.

An intermediate space $X$ of a Banach couple $\left(X_{0}, X_{1}\right)$ is called an interpolation space of $\left(X_{0}, X_{1}\right)$ if for any bounded linear operator from $\left(X_{0}, X_{1}\right)$ to itself, the restriction of $T$ to $X$ is a bounded operator from $X$ to itself. If in addition $\|T\|_{X \rightarrow X} \leq$ $\max _{i=0,1}\|T\|_{X_{i} \rightarrow X_{i}}$, the interpolation space $X$ is said to be exact.

For the actual construction of interpolation spaces of a given Banach couple $\left(X_{0}, X_{1}\right)$, different methods have been developed. The main methods are the complex and real interpolation methods. Thorin's proof of the Riesz-Thorin therorem constitutes the basis for the complex method of interpolation. It was introduced independently by A. P. Calderón, [5], [6] and J. L. Lions [13]. The real interpolation method was introduced by Lions and J. Peetre in [14] building on ideas in the proof of the Marcinkiewicz interpolation theorem. Our focus will be on the real method.

The core of the real interpolation method is Peetre's $K$-functional. We repeat its definition. Given a Banach couple $\left(X_{0}, X_{1}\right)$, the $K$-functional is defined by

$$
K\left(t, x ; X_{0}, X_{1}\right)=\inf _{x=x_{0}+x_{1}}\left(\left\|x_{0}\right\|_{X_{0}}+t\left\|x_{1}\right\|_{X_{1}}\right)
$$

for $x \in X_{0}+X_{1}$ and $t>0$. From the definition follows that for fixed $t>0$, $K\left(t, \cdot ; X_{0}, X_{1}\right)$ is a norm on $X_{0}+X_{1}$, and for fixed $x \in X_{0}+X_{1}, K\left(\cdot, x ; X_{0}, X_{1}\right)$ is a concave nonnegative function on $(0, \infty)$. 
On the basis of the $K$-functional, interpolation spaces can be constructed. The most common ones being the family of spaces $\left(X_{0}, X_{1}\right)_{\theta, q}, 0<\theta<1,1 \leq q \leq \infty$, given by

where

$$
\left(X_{0}, X_{1}\right)_{\theta, q}=\left\{x \in X_{0}+X_{1}:\|x\|_{\theta, q}<\infty\right\},
$$

$$
\|x\|_{\theta, q}=\left(\int_{0}^{\infty}\left(t^{-\theta} K\left(t, x ; X_{0}, X_{1}\right)\right)^{q} \frac{d t}{t}\right)^{\frac{1}{q}}, 1 \leq q<\infty,
$$

and

$$
\|x\|_{\theta, \infty}=\sup _{t>0} t^{-\theta} K\left(t, x ; X_{0}, X_{1}\right) .
$$

Further, with the $K$-functional introduced we can define a certain class of intermediate spaces. An intermediate space $X$ of a Banach couple $\left(X_{0}, X_{1}\right)$ is said to be $K$-monotone if

$$
K\left(\cdot, y ; X_{0}, X_{1}\right) \leq K\left(\cdot, x ; X_{0}, X_{1}\right), x \in X \Rightarrow y \in X,\|y\|_{X} \leq C\|x\|_{X}
$$

for some $C>0$.

Narrowing our focus to the couple $\left(L^{1}, L^{\infty}\right)$, we conclude the section by recalling some important results established by Calderón in [7]. The first is:

Theorem 1.3.1. Let $f, g \in L^{1}+L^{\infty}$ and suppose

$$
K\left(\cdot, g ; L^{1}, L^{\infty}\right) \leq K\left(\cdot, f ; L^{1}, L^{\infty}\right) .
$$

Then there exists a bounded linear operator $T$ from $\left(L^{1}, L^{\infty}\right)$ to $\left(L^{1}, L^{\infty}\right)$ with $\|T\|_{L^{1} \rightarrow L^{1}} \leq 1$ and $\|T\|_{L^{\infty} \rightarrow L^{\infty}} \leq 1$ such that $T f=g$.

From the Riesz convexity theorem, see e.g. Theorem IV.1.7 and Corollary IV.1.8 in [2, pp. 192-194], it follows that the operator $T$ of Theorem 1.3.1 also satisfies

$$
\|T\|_{L^{p} \rightarrow L^{p}} \leq\|T\|_{L^{1} \rightarrow L^{1}}^{1-\theta}\|T\|_{L^{\infty} \rightarrow L^{\infty}}^{\theta} \leq 1
$$

where $0<\theta<1$ and $1 / p=1-\theta$. Hence,

$$
\|T\|_{L^{p} \rightarrow L^{p}} \leq 1,1 \leq p \leq \infty .
$$

So for any $p \in[1, \infty]$, the inequality

$$
K\left(\cdot, g ; L^{1}, L^{\infty}\right) \leq K\left(\cdot, f ; L^{1}, L^{\infty}\right), f \in L^{p}
$$

gives that

$$
\|g\|_{L^{p}}=\|T f\|_{L^{p}} \leq\|T\|_{L^{p} \rightarrow L^{p}}\|f\|_{L^{p}} \leq\|f\|_{L^{p}} .
$$

Obviously, $\|g\|_{L^{p}} \leq\|f\|_{L^{p}}=\infty$ if $f \notin L^{p}$. From

$$
K\left(\cdot, g ; L^{1}, L^{\infty}\right) \leq K\left(\cdot, f ; L^{1}, L^{\infty}\right), f, g \in L^{1}+L^{\infty}
$$

therefore follows

$$
\|g\|_{L^{p}} \leq\|f\|_{L^{p}}, 1 \leq p \leq \infty .
$$

Going further, the following result formulated in [7] is a direct consequence of Theorem 1.3.1: 
Theorem 1.3.2. An intermediate space $X$ of $\left(L^{1}, L^{\infty}\right)$ is an exact interpolation space if and only if it is $K$-monotone with constant $C=1$.

By a resonant measure space, we denote a $\sigma$-finite measure space which is either (i) nonatomic or (ii) atomic with all atoms having equal measure. The final result of [7] which we recall is:

Theorem 1.3.3. Let $X$ be a Banach function space over a resonant measure space. Then $X$ is an exact interpolation space of $\left(L^{1}, L^{\infty}\right)$ if and only if $X$ is rearrangementinvariant.

For a definition of rearrangement-invariant Banach function spaces, we refer to Definition II.4.1 in [2, p. 59].

Restricting to a resonant measure space, Theorems 1.3.2 and 1.3.3 give that from

$$
K\left(\cdot, g ; L^{1}, L^{\infty}\right) \leq K\left(\cdot, f ; L^{1}, L^{\infty}\right), f, g \in L^{1}+L^{\infty}
$$

follows

$$
\|g\|_{X} \leq\|f\|_{X}
$$

for every rearrangement-invariant Banach function space $X$. 


\section{Summary of papers}

\subsection{Paper I: Discrete taut strings and real inter- polation}

In order to formulate the results of Paper I we introduce the notion of special directions:

Definition 2.1.1. The vectors

$$
v=\left\{\begin{array}{l} 
\pm e_{k}, k \in\{1, \ldots, n\} \\
\pm\left(e_{k}+e_{l}\right), k, l \in\{1, \ldots, n\}, k \neq l, \\
\pm\left(e_{k}-e_{l}\right), k, l \in\{1, \ldots, n\}, k \neq l
\end{array}\right.
$$

where $\left\{e_{i}\right\}_{i=1}^{n}$ is the standard basis of $\mathbb{R}^{n}$, will be referred to as the special directions in $\mathbb{R}^{n}$.

The main result of Paper I is the following characterization for the couple $\left(\ell^{1}, \ell^{\infty}\right)$ :

Theorem 2.1.1. A bounded, closed and convex set $\Omega \subset \mathbb{R}^{n}$ is invariant $K$-minimal with respect to $\left(\ell^{1}, \ell^{\infty}\right)$ if and only if $\Omega$ is a convex polytope where the affine hull of any face of $\Omega$ is a shifted subspace of $\mathbb{R}^{n}$ spanned by a basis consisting of special directions.

We recall that a convex polytope is a bounded, closed and convex set which is the intersection of a finite number of closed half-spaces. Moreover, given a convex set $S \subset \mathbb{R}^{n}$, a set $F \subset S$ is a face of $S$ if either $F=\emptyset$ or $F=S$ or if there exists a supporting hyperplane $H$ of $S$ such that $F=S \cap H$. For background material on convex polytopes, see e.g. [9].

The proof, known to us, of the necessity part of Theorem 2.1.1 is rather involved. An important notion when deriving Theorem 2.1.1 is the special cone property which we now introduce:

Definition 2.1.2. Let $\Omega \subset \mathbb{R}^{n}$ be closed and convex. For $x \in \Omega$, take all special directions $v$ such that $x+\beta v \in \Omega$ for sufficiently small $\beta>0$. Let $S_{x}$ denote the set of all such special directions at $x \in \Omega$. Further, let

$$
K_{x}=\left\{y \in \mathbb{R}^{n}: y=\sum_{v \in S_{x}} \alpha_{v} v, \alpha_{v} \geq 0\right\}
$$


be the convex cone generated by the special directions in $S_{x}$. We say that $\Omega$ has the special cone property if

$$
\left(x+K_{x}\right) \cap \Omega=\Omega
$$

for each $x \in \Omega$.

The following sufficient condition in terms of the special cone property can be given:

Theorem 2.1.2. Let $\Omega \subset \mathbb{R}^{n}$ be a closed and convex set that has the special cone property. Then $\Omega$ is an invariant $K$-minimal set with respect to $\left(\ell^{1}, \ell^{\infty}\right)$.

Note that the set $\Omega$ in Theorem 2.1.2 needs not to be bounded. Besides being used in the proof of Theorem 2.1.1, this theorem is useful when constructing concrete examples of invariant $K$-minimal sets with respect to $\left(\ell^{1}, \ell^{\infty}\right)$.

Closely connected to invariant $K$-minimal sets with respect to $\left(\ell^{1}, \ell^{\infty}\right)$ are so called invariant $\varphi$-minimal sets. Given $z=\left(z_{1}, \ldots, z_{n}\right) \in \mathbb{R}^{n}$, let $z^{\downarrow} \in \mathbb{R}^{n}$ denote the vector with the elements of $z$ sorted in decreasing order. Note that this is not the decreasing rearrangement of the modulus of the elements of $z$. An example: if $z=(2,-8,9)$ then $z^{\downarrow}=(9,2,-8)$. With this notation introduced, we recall the classical majorization inequality of Hardy, Littlewood and Pólya established in [10]:

$$
\sum_{i=1}^{n} \varphi\left(x_{i}\right) \leq \sum_{i=1}^{n} \varphi\left(y_{i}\right)
$$

holds for $x, y \in \mathbb{R}^{n}$ and all convex functions $\varphi: \mathbb{R} \rightarrow \mathbb{R}$ if and only if

$$
\sum_{i=1}^{k} x_{i}^{\downarrow} \leq \sum_{i=1}^{k} y_{i}^{\downarrow}, k=1, \ldots, n-1,
$$

and

$$
\sum_{i=1}^{n} x_{i}=\sum_{i=1}^{n} y_{i}
$$

If (2.1) and (2.2) hold we say that $x$ is majorized by $y$. Since its appearance, the Hardy-Littlewood-Pólya majorization inequality has found widespread applications in e.g. statistics, physics and economics. For a detailed account on majorization and its applications, see [17].

With the Hardy-Littlewood-Pólya majorization inequality in mind, we introduce the notion of invariant $\varphi$-minimal sets:

Definition 2.1.3. A set $\Omega \subset \mathbb{R}^{n}$ will be denoted invariant $\varphi$-minimal if for every element $a \in \mathbb{R}^{n}$ there exists an element $x_{*, a} \in \Omega$ such that

$$
\sum_{i=1}^{n} \varphi\left(x_{*, a, i}-a_{i}\right) \leq \sum_{i=1}^{n} \varphi\left(x_{i}-a_{i}\right)
$$

holds for every $x \in \Omega$ and all convex functions $\varphi: \mathbb{R} \rightarrow \mathbb{R}$. 
It is instructive to compare Definition 2.1.3 with the following characterization of invariant $K$-minimal sets in terms of a special class of convex functions:

Proposition 2.1.1. The set $\Omega \subset \mathbb{R}^{n}$ is an invariant $K$-minimal set with respect to $\left(\ell^{1}, \ell^{\infty}\right)$ if and only if for every $a \in \mathbb{R}^{n}$ there exists an element $x_{*, a} \in \Omega$ satisfying

$$
\sum_{i=1}^{n} \varphi\left(x_{*, a, i}-a_{i}\right) \leq \sum_{i=1}^{n} \varphi\left(x_{i}-a_{i}\right),
$$

for all $x \in \Omega$ and all even and convex functions $\varphi: \mathbb{R} \rightarrow \mathbb{R}$.

The precise connection between invariant $\varphi$-minimal sets and invariant $K$-minimal sets with respect to $\left(\ell^{1}, \ell^{\infty}\right)$ is given by

Theorem 2.1.3. Let $\Omega \subset \mathbb{R}^{n}$ be a bounded, closed and convex set. Then $\Omega$ is invariant $\varphi$-minimal if and only if (i) $\Omega \subset H=\left\{y \in \mathbb{R}^{n}: \sum_{i=1}^{n} y_{i}=C\right\}$ for some $C \in \mathbb{R}$ and (ii) $\Omega$ is invariant $K$-minimal with respect to $\left(\ell^{1}, \ell^{\infty}\right)$.

So, an invariant $K$-minimal set with respect to $\left(\ell^{1}, \ell^{\infty}\right)$ which is embedded in a hyperplane $H=\left\{y \in \mathbb{R}^{n}: \sum_{i=1}^{n} y_{i}=C\right\}$ is in addition invariant $\varphi$-minimal.

Having Theorem 2.1.3 at our disposal, it is possible to give the following analogue of Theorem 2.1.1 for invariant $\varphi$-minimal sets:

Theorem 2.1.4. A bounded, closed and convex set $\Omega \subset \mathbb{R}^{n}$ is invariant $\varphi$-minimal if and only if $\Omega$ is a convex polytope where the affine hull of any face of $\Omega$ is a shifted subspace of $\mathbb{R}^{n}$ spanned by a basis consisting of special directions of the type $e_{i}-e_{j}$, $i \neq j$.

Remark 2.1.1. Note the restriction to special directions of the type $e_{i}-e_{j}, i \neq j$, in Theorem 2.1.4.

\subsection{Paper II: Invariant $K$-minimal sets in the dis- crete and continuous settings}

This section is divided into three subsections covering the main results of Paper II.

\subsubsection{Divergence of flows on graphs}

We consider here a particular example of an invariant $K$-minimal set with respect to $\left(\ell^{1}, \ell^{\infty}\right)$. This example is constructed through the sufficient condition established in Theorem 2.1.2 and can be viewed as a generalization of the taut string problem with fixed ends.

Consider a finite directed graph $(V, E)$ with $n$ vertices $v_{1}, \ldots, v_{n} \in V$ and $m$ edges $\left(v_{i_{j}}, v_{k_{j}}\right) \in E, j=1, \ldots, m$. We make the convention that the edge $\left(v_{i}, v_{k}\right)$ is directed from $v_{i}$ to $v_{k}$. Let $S_{V}$ denote the $n$-dimensional space of real-valued 
functions defined on $V$. Analogously, $S_{E}$ denotes the $m$-dimensional space of realvalued functions defined on $E$. The divergence operator div $: S_{E} \rightarrow S_{V}$ is defined by

$$
(\operatorname{div} f)\left(v_{k}\right)=\sum_{i:\left(v_{i}, v_{k}\right) \in E} f\left(v_{i}, v_{k}\right)-\sum_{j:\left(v_{k}, v_{j}\right) \in E} f\left(v_{k}, v_{j}\right) .
$$

Thinking of the elements of $S_{E}$ as flows on the graph $(V, E)$ gives the interpretation of the divergence at a vertex as the sum of all incoming flows minus the sum of all outgoing flows. See Figure 2.1 for an illustration.

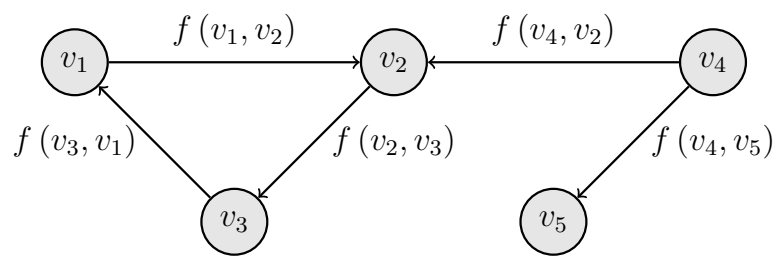

Figure 2.1: Example of a graph with flows.

Given $F, G \in S_{E}$ with $F \leq G$, i.e. $F\left(v_{i}, v_{k}\right) \leq G\left(v_{i}, v_{k}\right)$ for every $\left(v_{i}, v_{k}\right) \in E$, consider the rectangular domain $R_{F, G}$ in $S_{E}$ :

$$
R_{F, G}=\left\{f \in S_{E}: F \leq f \leq G\right\} .
$$

We establish the following result:

Theorem 2.2.1. The set $\operatorname{div}\left(R_{F, G}\right)$, i.e. the image of $R_{F, G}$ under the divergence operator div, is an invariant $K$-minimal set with respect to $\left(\ell^{1}, \ell^{\infty}\right)$ on $S_{V}$.

Remark 2.2.1. It can be shown that $\operatorname{div}\left(R_{F, G}\right)$ in addition is invariant $\varphi$-minimal, i.e. given $a \in S_{V}$ there exists an element $x_{*, a} \in \operatorname{div}\left(R_{F, G}\right)$ such that

$$
\sum_{v \in V} \varphi\left(x_{*, a}(v)-a(v)\right) \leq \sum_{v \in V} \varphi(x(v)-a(v))
$$

holds for every $x \in \operatorname{div}\left(R_{F, G}\right)$ and every convex function $\varphi: \mathbb{R} \rightarrow \mathbb{R}$.

Let us briefly discuss a possible interpretation of Theorem 2.2.1 and Remark 2.2.1. Suppose the directed graph $(V, E)$ represents a network where water flows through pipes (edges) to different locations (vertices). Each pipe $e \in E$ has a given capacity $G(e) \geq 0$. The network is running in normal condition and then one or several pipes becomes broken with reduced or no capacity. This might affect incoming and/or outgoing flow at some locations. Recall that the divergence at a location is the difference between incoming and outgoing flow. An increasing divergence at a location can be thought of as a flooding whereas a decreasing divergence at a location can be thought of as a drought. Let $G^{\prime}$ denote the capacity of the broken network. Suppose the divergence in the network during normal conditions is given by $a \in S_{V}$. By Theorem 2.2.1 and Remark 2.2.1, we know that it is possible to 
modify the flows in the network such that the resulting divergence $x_{*, a} \in \operatorname{div}\left(R_{0, G^{\prime}}\right)$ satisfies

$$
\sum_{v \in V} \varphi\left(x_{*, a}(v)-a(v)\right)=\inf _{x \in \operatorname{div}\left(R_{0, G^{\prime}}\right)} \sum_{v \in V} \varphi(x(v)-a(v))
$$

for all convex functions $\varphi: \mathbb{R} \rightarrow \mathbb{R}$. This can be viewed as minimizing the total impact of the broken pipes in the network or in other words optimally adjust the flows to the new network conditions.

The above approach of optimal management of a network under changing conditions seems to be useful in diverse applications. For an example in image processing, see $[20]$.

\subsubsection{Algorithms}

When considering applications of invariant $K$-minimal sets with respect to $\left(\ell^{1}, \ell^{\infty}\right)$, for instance the example of the preceding subsection, it is important to compute the element $x_{*, a}$ which satisfies

$$
K\left(t, x_{*, a}-a ; \ell^{1}, \ell^{\infty}\right) \leq K\left(t, x-a ; \ell^{1}, \ell^{\infty}\right)
$$

for every $x \in \Omega$ and $t>0$. As the $\ell^{2}$-norm can be used, there are a lot of potential algorithms available for this task. However, the structure of invariant $K$-minimal sets can be rather complicated. We will therefore present an algorithm which is tailored for invariant $K$-minimal sets. It is in principal based on $\ell^{2}$-minimization along search directions given by the special directions. Before formulating the algorithm in detail, we introduce some notation.

Suppose $\Omega \subset \mathbb{R}^{n}$ is closed and convex and satisfies the special cone property (recall Definition 2.1.1). Fix $a, v \in \mathbb{R}^{n}$. Let $P_{a, v}: \Omega \rightarrow \Omega$ denote the operator defined by the conditions

$$
P_{a, v}(x) \in\{x+t v: t \in \mathbb{R}\} \cap \Omega
$$

and

$$
\left\|P_{a, v}(x)-a\right\|_{\ell^{2}}=\inf _{u \in\{x+t v: t \in \mathbb{R}\} \cap \Omega}\|u-a\|_{\ell^{2}} .
$$

So $P_{a, v}$ maps $x \in \Omega$ onto the element of best approximation of $a$, with respect to $\ell^{2}$-norm, on the line segment $\{x+t v: t \in \mathbb{R}\} \cap \Omega$.

Given $x \in \Omega$, let $S_{x}$ denote the set of all special directions $v$ such that $x+\beta v \in \Omega$ for small enough $\beta>0$. Choose a special direction $v_{x} \in S_{x}$ such that

$$
\left\|P_{a, v_{x}}(x)-a\right\|_{\ell^{2}}=\min _{v \in S_{x}}\left\|P_{a, v}(x)-a\right\|_{\ell^{2}} .
$$

If there are several special directions $v$ that fulfill (2.3) we can choose $v_{x}$ to be any of them. The operator $P_{a}: \Omega \rightarrow \Omega$ is now defined according to $P_{a}(x)=P_{a, v_{x}}(x)$. For an illustration, see Figure 2.2.

We have now introduced the necessary notation and can formulate the following result: 


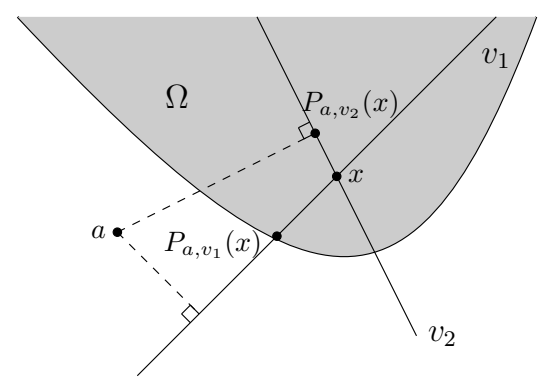

Figure 2.2: Illustration of projections along special directions, in this case $P_{a}(x)=$ $P_{a, v_{1}}(x)$.

Theorem 2.2.2. Let $\Omega \subset \mathbb{R}^{n}$ be closed and convex and satisfy the special cone property. Fix $a \in \mathbb{R}^{n}$. Choose any $x_{0} \in \Omega$. Let $x_{n}=P_{a}\left(x_{n-1}\right), n \in \mathbb{N}$. Then $x_{n} \rightarrow x_{*, a}$ as $n \rightarrow \infty$.

The algorithm takes any given starting point $x_{0} \in \Omega$ and then update $x_{n}=$ $P_{a}\left(x_{n-1}\right), n \in \mathbb{N}$. Theorem 2.2.2 shows that the resulting sequence $\left\{x_{n}\right\}_{n \in \mathbb{N}}$ converges to the nearest element $x_{*, a}$ in $\Omega$ of $a$.

The main challenge in the algorithm is the determination of the special directions in $S_{x}$ for a given $x \in \Omega$. In applications one can try to take advantage of the specific structure of the set $\Omega$ and thereby specialize the algorithm for the problem under consideration. For an example, see [20] where a special version of the algorithm is applied to the Rudin-Osher-Fatemi image denoising model.

Considering the taut string problem with fixed ends, a different algorithm that computes the element $x_{*, a}$ in a finite number of steps can be given, see Papers III and IV.

\subsubsection{Continuous setting}

We now turn to the continuous setting and consider invariant $K$-minimal sets with respect to the couple $\left(L^{1}\left([0,1]^{m}\right), L^{\infty}\left([0,1]^{m}\right)\right)$. Real $L^{p}$-spaces are assumed everywhere in this subsection. Our tool for constructing concrete invariant $K$-minimal sets in this setting is a limiting procedure applied to families of finite-dimensional invariant $K$-minimal sets with certain properties. Before making this precise, we need to introduce some notation.

First, the following space of piecewise constant functions on $[0,1]^{m}$ is introduced:

Definition 2.2.1. Let $S_{n}\left([0,1]^{m}\right)$ denote the $n^{m}$-dimensional space of piecewise constant functions on $[0,1]^{m}$ given by

$$
S_{n}\left([0,1]^{m}\right)=\left\{g: g=\sum_{i_{1}, \ldots, i_{m}=1}^{n} c_{i_{1} \ldots i_{m}} \chi_{\Gamma_{i_{1} \ldots i_{m}}^{n}}, c_{i_{1} \ldots i_{m}} \in \mathbb{R}\right\}
$$

where $\Gamma_{i_{1} \ldots i_{m}}^{n}:=\left[\frac{i_{1}-1}{n}, \frac{i_{1}}{n}\right) \times \ldots \times\left[\frac{i_{m}-1}{n}, \frac{i_{m}}{n}\right), i_{1}, \ldots, i_{m}=1, \ldots, n$, and $\chi_{\Gamma_{i_{1} \ldots i_{m}}^{n}}$ is the characteristic function of $\Gamma_{i_{1} \ldots i_{m}}^{n}$. 
For each $g=\sum_{i_{1}, \ldots, i_{m}=1}^{n} c_{i_{1} \ldots i_{m}} \chi_{\Gamma_{i_{1} \ldots i_{m}}^{n}} \in S_{n}\left([0,1]^{m}\right)$, we have a corresponding uniquely determined coefficient vector $c=\left(c_{1 \ldots 1}, \ldots, c_{n \ldots n}\right) \in \mathbb{R}^{n^{m}}$. Based on this observation, we introduce:

Definition 2.2.2. Given $\Gamma \subset S_{n}\left([0,1]^{m}\right)$, we denote by $\widetilde{\Gamma}$ the set in $\mathbb{R}^{n^{m}}$ which consists of all coefficient vectors corresponding to the elements in $\Gamma$.

Let $\mathcal{M}$ denote the set of real-valued Lebesgue measurable functions on $[0,1]^{m}$. We define the averaging operator $P_{n}: \mathcal{M} \rightarrow S_{n}$ by

$$
P_{n} f=\sum_{i_{1}, \ldots, i_{m}=1}^{n} \frac{1}{\left(\frac{1}{n}\right)^{m}}\left(\int_{\Gamma_{i_{1} \ldots i_{m}}^{n}} f(s) d s\right) \chi_{\Gamma_{i_{1} \ldots i_{m}}^{n}} .
$$

The necessary notation is now introduced in order to present the following sufficient condition for a set $\Omega \subset L^{1}\left([0,1]^{m}\right)$ to be invariant $K$-minimal with respect to $\left(L^{1}\left([0,1]^{m}\right), L^{\infty}\left([0,1]^{m}\right)\right)$ :

Theorem 2.2.3. Let $\left\{\Omega_{2^{n}}\right\}_{n \in \mathbb{N}}$ be a family of sets such that for each $n \in \mathbb{N}$ :

(i) $\Omega_{2^{n}}$ is a closed and convex subset of $S_{2^{n}}\left([0,1]^{m}\right)$ and $P_{2^{n}}: \Omega_{2^{n+1}} \rightarrow \Omega_{2^{n}}$,

(ii) the set of coefficient vectors $\widetilde{\Omega}_{2^{n}}$ is invariant $K$-minimal with respect to $\left(\ell^{1}, \ell^{\infty}\right)$ on $\mathbb{R}^{2^{n m}}$.

Then the set

$$
\Omega=\left\{h \in L^{1}\left([0,1]^{m}\right): P_{2^{n}} h \in \Omega_{2^{n}}, \forall n \in \mathbb{N}\right\}
$$

is invariant $K$-minimal with respect to $\left(L^{1}\left([0,1]^{m}\right), L^{\infty}\left([0,1]^{m}\right)\right)$.

Invariant $\varphi$-minimal sets in the setting of $L^{1}\left([0,1]^{m}\right)$ are now introduced, compare with Definition 2.1.3.

Definition 2.2.3. A set $\Omega \subset L^{1}\left([0,1]^{m}\right)$ is called invariant $\varphi$-minimal if for every element $f \in L^{1}\left([0,1]^{m}\right)$, there exists an element $g_{f}^{*} \in \Omega$ such that

$$
\int_{[0,1]^{m}} \varphi\left(g_{f}^{*}(x)-f(x)\right) d x \leq \int_{[0,1]^{m}} \varphi(g(x)-f(x)) d x
$$

for every $g \in \Omega$ and every convex function $\varphi: \mathbb{R} \rightarrow \mathbb{R}$.

The following sufficient condition for invariant $\varphi$-minimal sets in $L^{1}\left([0,1]^{m}\right)$ holds:

Theorem 2.2.4. Let $\left\{\Omega_{2^{n}}\right\}_{n \in \mathbb{N}}$ be a family of sets such that for each $n \in \mathbb{N}$ :

(i) $\Omega_{2^{n}}$ is a closed and convex subset of $S_{2^{n}}\left([0,1]^{m}\right), P_{2^{n}}: \Omega_{2^{n+1}} \rightarrow \Omega_{2^{n}}$ and $\int_{[0,1]^{m}} g(x) d x=C, \forall g \in \Omega_{2^{n}}$, for some $C \in \mathbb{R}$,

(ii) the set of coefficient vectors $\widetilde{\Omega}_{2^{n}}$ has the special cone property. 
Then the set

$$
\Omega=\left\{h \in L^{1}\left([0,1]^{m}\right): P_{2^{n}} h \in \Omega_{2^{n}}, \forall n \in \mathbb{N}\right\}
$$

is invariant $\varphi$-minimal.

The proofs of Theorems 2.2.3 and 2.2.4 both use uniform boundedness in $L^{1}$ and uniform integrability combined with Mazur's lemma. However, since the functional $\int \varphi(\cdot)$ in general is not a norm on $L^{1}$ we need to use some additional tools in the proof of Theorem 2.2.4. Among them an inequality for convex functions originally formulated by Hardy, Littlewood and Pólya in [10].

With Theorem 2.2.3 at our disposal we will now provide two examples of invariant $K$-minimal sets in $L^{1}\left([0,1]^{m}\right)$.

First, an analogue of Theorem 1.2.1 for an infinite-dimensional version of the taut string problem is considered. Let $C[0,1]$ and $A C[0,1]$ denote the spaces of continuous respectively absolutely continuous functions on $[0,1]$.

Theorem 2.2.5. Let $F, G \in C[0,1]$ with $F \leq G$. Then the set

$$
\Lambda:=\left\{h \in L^{1}[0,1]: \exists H \in A C[0,1], F \leq H \leq G, h=H^{\prime} \text { a.e. on }[0,1]\right\}
$$

is invariant $K$-minimal with respect to $\left(L^{1}[0,1], L^{\infty}[0,1]\right)$.

The proof of Theorem 2.2.5 starts by showing that the family of sets $\left\{\Omega_{2^{n}}\right\}_{n \in \mathbb{N}}$ given by

$$
\begin{aligned}
& \Omega_{2^{n}}=\left\{\widehat{h} \in S_{2^{n}}[0,1]: \exists D \in[F(0), G(0)]\right. \text { s.t. } \\
&\left.F\left(\frac{k}{2^{n}}\right) \leq D+\int_{0}^{\frac{k}{2^{n}}} \widehat{h}(s) d s \leq G\left(\frac{k}{2^{n}}\right), k=0,1, \ldots, 2^{n}\right\}
\end{aligned}
$$

satisfies the conditions of Theorem 2.2.3. The second part of the proof is then devoted to show $\Lambda=\left\{h \in L^{1}[0,1]: P_{2^{n}} h \in \Omega_{2^{n}}\right\}$.

Remark 2.2.2. If $F(0)=G(0)$ and $F(1)=G(1)$ it can be shown that the family of sets $\left\{\Omega_{2^{n}}\right\}_{n \in \mathbb{N}}$ satisfies the conditions of Theorem 2.2.4. So, in this case the set $\Lambda$ is in addition invariant $\varphi$-minimal, i.e. given $f \in L^{1}[0,1]$ there exists $g_{*, f} \in \Lambda$ such that

$$
\int_{0}^{1} \varphi\left(g_{*, f}(x)-f(x)\right) d x \leq \int_{0}^{1} \varphi(g(x)-f(x)) d x
$$

for every $g \in \Lambda$ and all convex functions $\varphi: \mathbb{R} \rightarrow \mathbb{R}$.

Remark 2.2.3. Let $F, G$ be continuous piecewise linear functions on $[0,1]$ with nodes in $\frac{i}{m}, i=0,1, \ldots, m, m \in \mathbb{N}$, where $G\left(\frac{i}{m}\right)=F\left(\frac{i}{m}\right)+\alpha, i=1, \ldots, m-1$, for some $\alpha>0$ and $F(0)=G(0), F(1)=G(1)$. In this case, the result of Remark 2.2.2 was known before, see [23, Theorem 4.35, $p$. 141]. 
The second example of an invariant $K$-minimal set in $L^{1}\left([0,1]^{m}\right)$ is related to an infinite-dimensional analogue of divergence of flows on the graph (recall Theorem 2.2.1). Denote the space of $m$-dimensional smooth vector fields with support in $(0,1)^{m}$ by $C_{c}^{\infty}\left((0,1)^{m}, \mathbb{R}^{m}\right)$. Define $L^{\infty}\left([0,1]^{m}, \mathbb{R}^{m}\right)$ as the space of all $m$ dimensional Lebesgue measurable vector fields $\mathbf{F}=\left(F_{1}, \ldots, F_{m}\right):[0,1]^{m} \rightarrow \mathbb{R}^{m}$ such that $\|\mathbf{F}\|_{L^{\infty}}:=\max _{1 \leq i \leq m}\left\{\left\|F_{i}\right\|_{L^{\infty}}\right\}<\infty$. With this notation introduced, we can formulate:

Theorem 2.2.6. Let

$$
M:=\left\{\mathbf{F} \in C_{c}^{\infty}\left((0,1)^{m}, \mathbb{R}^{m}\right):\|\mathbf{F}\|_{L^{\infty}} \leq 1\right\}
$$

and

$$
\operatorname{div} M:=\left\{f \in C_{c}^{\infty}\left((0,1)^{m}\right): f=\operatorname{div} \mathbf{F}, \mathbf{F} \in M\right\} .
$$

Then the closure of $\operatorname{div} M$ in $L^{1}$, denoted $\overline{\operatorname{div} M}$, is an invariant $K$-minimal set with respect to $\left(L^{1}\left([0,1]^{m}\right), L^{\infty}\left([0,1]^{m}\right)\right)$.

The proof of Theorem 2.2.6 starts, as the proof of Theorem 2.2.5, by constructing a family of sets $\left\{\Omega_{2^{n}}\right\}_{n \in \mathbb{N}}$ which satisfies the conditions of Theorem 2.2.3. We show below the construction of $\left\{\Omega_{2^{n}}\right\}_{n \in \mathbb{N}}$ for $m=2$ (the case $m>2$ is similar). Let

$$
\Omega_{2^{n}}=\left\{g \in S_{2^{n}}\left([0,1]^{2}\right): g=g_{1}+g_{2}, g_{1} \in \Omega_{2^{n}}^{1}, g_{2} \in \Omega_{2^{n}}^{2}\right\}
$$

where

$$
\Omega_{2^{n}}^{1}=\left\{g_{1} \in S_{2^{n}}\left([0,1]^{2}\right): \sup _{x \in[0,1]}\left|\int_{0}^{x} g_{1}(s, y) d s\right| \leq 1, \int_{0}^{1} g_{1}(s, y) d s=0\right\}
$$

and

$$
\Omega_{2^{n}}^{2}=\left\{g_{2} \in S_{2^{n}}\left([0,1]^{2}\right): \sup _{y \in[0,1]}\left|\int_{0}^{y} g_{2}(x, t) d t\right| \leq 1, \int_{0}^{1} g_{2}(x, t) d s=0\right\} .
$$

A key step in the process of showing that $\left\{\Omega_{2^{n}}\right\}_{n \in \mathbb{N}}$ satisfies the conditions of Theorem 2.2.3 is to interpret $\Omega_{2^{n}}$ on a certain graph. However, we will not go into details here but refer to Paper II.

The remaining part of the proof is then to show the characterization

$$
\overline{\operatorname{div} M}=\Omega:=\left\{h \in L^{1}\left([0,1]^{2}\right): P_{2^{n}} h \in \Omega_{2^{n}}, \forall n \in \mathbb{N}\right\} .
$$

While $\overline{\operatorname{div} M} \subset \Omega$ is fairly straightforward to establish, the $\operatorname{direction} \overline{\operatorname{div} M} \supset \Omega$ requires more efforts. In this direction, the main problem is to establish $\Omega_{2^{n}} \subset \overline{\operatorname{div} M}$ for every $n \in \mathbb{N}$.

Remark 2.2.4. It can be shown that the family of sets $\left\{\Omega_{2^{n}}\right\}_{n \in \mathbb{N}}$ defined by (2.4) also satisfies the conditions of Theorem 2.2.4. We can then conclude that $\overline{\operatorname{div} M}$ in addition is invariant $\varphi$-minimal, i.e. given $f \in L^{1}\left([0,1]^{m}\right)$ there is an element $g_{*, f} \in \overline{\operatorname{div} M}$ such that

$$
\int_{[0,1]^{m}} \varphi\left(g_{*, f}(x)-f(x)\right) d x \leq \int_{[0,1]^{m}} \varphi(g(x)-f(x)) d x
$$

holds for every $g \in \overline{\operatorname{div} M}$ and all convex functions $\varphi: \mathbb{R} \rightarrow \mathbb{R}$. In [23], the possibility to have such result under some additional conditions was pointed out, see Remark 4.47 on $p .150$. 


\subsection{Paper III: Real-time communication systems based on taut strings}

Paper III studies an application in real-time communication where a taut string problem, and therefore invariant $K$-minimal sets, appear. The starting point for our work in this field was the investigations of optimal buffer management strategies in [19]. For related work in video smoothing, see [22].

Consider a real-time communication system where a sender transmits information to a receiver through a channel with time-dependent capacity. In order to allow for information that cannot be immediately transmitted, the sender has a buffer that can store some amount of information for transmission later on when the channel capacity is expected to be higher. One typical example in this setting is live broadcasting of video over the Internet. A problem then emerges concerning how to use the buffer optimally throughout the sending period with respect to the communication quality. We will now try to make this rather vague problem more precise.

The basic communication system is illustrated in Figure 2.3. We consider a send-

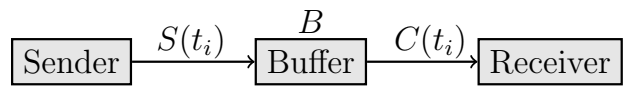

Figure 2.3: The basic commmunication system.

ing period of discrete time instances $\left\{t_{1}, t_{2}, \ldots, t_{n}\right\}$ which are uniformly distributed, i.e $t_{i+1}-t_{i}=\Delta>0$ for $i=1, . ., n-1$. By $S\left(t_{i}\right)$, measured in bits, we denote the sender production at $t_{i}$. $C\left(t_{i}\right)$, measured in bits, is the channel capacity at $t_{i}$ and is assumed to be known at all time instances. Finally, $B$, measured in bits, is the buffer capacity which is independent of time.

All bits of $S\left(t_{i}\right)$ are assumed to pass through the buffer before entering the channel. The buffer works according to the "First In, First Out" (FIFO) queuing principle: the saved bits are sent through the channel to the receiver in the same order as they entered the buffer.

The set $\Omega$ of admissible buffer management strategies is given by

$$
\begin{gathered}
\Omega=\left\{S \in \mathbb{R}^{n}: \sum_{i=1}^{j} C\left(t_{i}\right) \leq \sum_{i=1}^{j} S\left(t_{i}\right) \leq \sum_{i=1}^{j} C\left(t_{i}\right)+B, j=1, \ldots, n-1\right. \\
\left.\sum_{i=1}^{n} S\left(t_{i}\right)=\sum_{i=1}^{n} C\left(t_{i}\right)+B\right\} .
\end{gathered}
$$

The conditions of $\Omega$ imply that for $j=1, \ldots, n-1$, the cumulative strategy $\sum_{i=1}^{j} S\left(t_{i}\right)$ should be in between the cumulative channel capacity and the cumulative channel capacity plus the buffer capacity. Further, at the end of the sending period, i.e. at $t_{n}$, we should have used all available channel capacity and buffer capacity.

The information loss or distortion during the sending period $\left\{t_{1}, t_{2}, \ldots, t_{n}\right\}$ is 
measured by the quantity

$$
\sum_{i=1}^{n} \varphi\left(S\left(t_{i}\right)\right)
$$

where $\varphi: \mathbb{R} \rightarrow \mathbb{R}$ typically is a decreasing convex function. For the moment, let us consider $\varphi$ to be fixed.

The resulting buffer management problem is to find a buffer management strategy $S_{*} \in \Omega$ such that

$$
\sum_{i=1}^{n} \varphi\left(S_{*}\left(t_{i}\right)\right)=\inf _{S \in \Omega} \sum_{i=1}^{n} \varphi\left(S\left(t_{i}\right)\right) .
$$

Now, it can be shown that the set $\Omega$ is an invariant $\varphi$-minimal set in $\mathbb{R}^{n}$. In fact, $\Omega$ corresponds to a taut string problem with fixed ends. So there is a unique buffer management strategy $S_{*} \in \Omega$ that solves (2.5) for all convex functions $\varphi$. Hence, we have diverse characterizations of the optimal buffer management strategy $S_{*}$. Further, in communication theory the choice of $\varphi$ is typically an exponential function. But because of its independence of $\varphi$, it possible to apply algorithms based on the choices $\varphi(x)=x^{2}$, as the algorithm of Subsection 2.2.2, or $\varphi(x)=\sqrt{1+x^{2}}$, which is done in Paper III, for computing $S_{*}$.

\subsection{Paper IV: Energy of taut strings accompany- ing Wiener process}

In Paper IV, an application of non-classical taut strings to the Wiener process (standard one-dimensional Brownian motion) is considered. For background material on the Wiener process, see e.g. Chapter 2 in [11].

Given a trajectory of a Wiener process $W$ on the interval $[0, T]$, we construct a uniform piecewise linear approximation of it. More precisely, $W$ is evaluated at $\frac{i T}{N}, i=0,1, \ldots, N$, for some $N \in \mathbb{N}$ and interpolated linearly in between these nodes. Given $r, T>0$, let $F$ and $G$ be the continuous piecewise linear functions on $[0, T]$ with nodes in $\frac{i T}{N}, i=0,1, \ldots, N$, where $F(0)=G(0)=W(0)=0, F\left(\frac{i T}{N}\right)=$ $W\left(\frac{i T}{N}\right)-r$ and $G\left(\frac{i T}{N}\right)=W\left(\frac{i T}{N}\right)+r, i=1, \ldots, N$. Further, let $\Gamma_{F, G}$ denote the set of all continuous piecewise linear functions $f$ on $[0, T]$ with nodes in $\frac{i T}{N}, i=0,1, \ldots, N$, and which satisfies $F \leq f \leq G$. Hence, a function $f \in \Gamma_{F, G}$ will start at 0 , as the trajectory of the Wiener process, and for $t \in(0, T]$ be at most a distance $r$ from the piecewise linear approximation of the trajectory of the Wiener process.

With the above notation introduced, consider the problem:

Problem 2.4.1. Find the function $f_{*} \in \Gamma_{F, G}$ that satisfies

$$
\int_{0}^{T} \sqrt{1+f_{*}^{\prime}(t)^{2}} d t=\inf _{f \in \Gamma_{F, G}} \int_{0}^{T} \sqrt{1+f^{\prime}(t)^{2}} d t .
$$

This is a taut string problem with a free end at $t=T$. For an illustration, see Figure 2.4. The set

$$
\Omega=\left\{u \in \mathbb{R}^{N}: u_{i}=\frac{f(i T / N)-f((i-1) T / N)}{T / N}, f \in \Gamma_{F, G}\right\}
$$




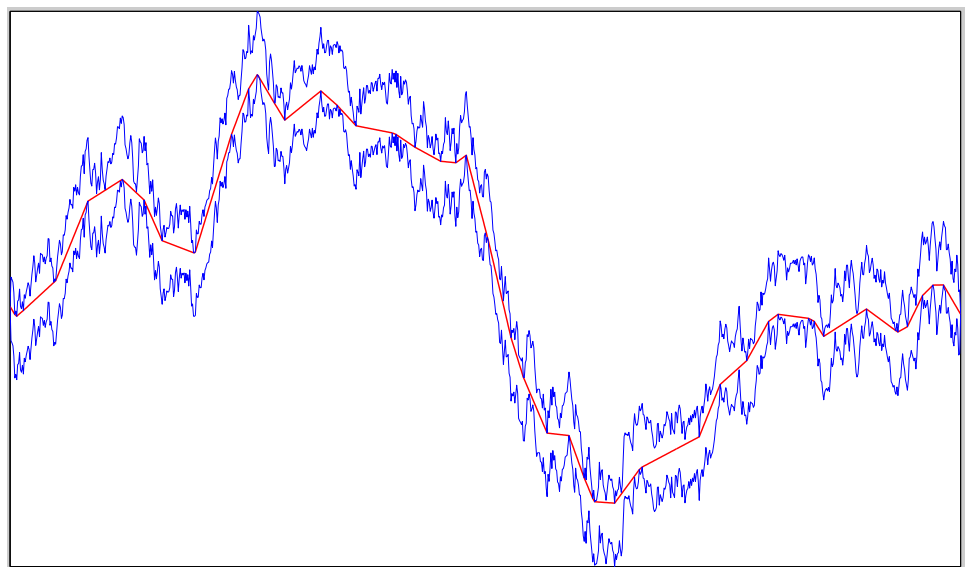

Figure 2.4: Taut string (red) inside the corridor $\Gamma_{F, G}$.

is by Theorem 1.2.1 an invariant $K$-minimal set with respect to $\left(\ell^{1}, \ell^{\infty}\right)$ and it follows that the element $u_{*} \in \Omega$ with

$$
u_{*, i}=\frac{f_{*}(i T / N)-f_{*}((i-1) T / N)}{T / N}, i=1, \ldots, n,
$$

i.e. the vector in $\Omega$ corresponding to the piecewise constant derivative of the taut string $f_{*} \in \Gamma_{F, G}$, has minimal $K$-functional in $\Omega$. This gives in turn that the taut string $f_{*}$ also satisfies

$$
\int_{0}^{T} f_{*}^{\prime}(t)^{2} d t=\inf _{f \in \Gamma_{F, G}} \int_{0}^{T} f^{\prime}(t)^{2} d t
$$

So, $f_{*}$ has minimal energy among the functions in $\Gamma_{F, G}$.

Consider now the quantity

$$
r\left(\frac{1}{T} \int_{0}^{T} f_{*}^{\prime}(t)^{2} d t\right)^{1 / 2}
$$

i.e. the distance $r$ times the square root of the average power of the taut string. Numerical simulations conducted at the National Supercomputer Centre at Linköping University indicate that the mean of this quantity, considering a large sample of independent trajectories of the Wiener process, converges towards a constant $\mathcal{C} \approx 0.63$ when $T \rightarrow \infty$ and the step size $T / N$ is small enough. These simulations are based on an algorithm for computing finite-dimensional taut strings. We now present a rigorous interpretation in the continuous setting of the numerical simulations.

Let $W$ be a Wiener process. Given $r, T>0$, let

$$
\Lambda_{r, T}=\left\{f \in A C[0, T]: \sup _{t \in[0, T]}|f(t)-W(t)| \leq r, f(0)=W(0)=0\right\}
$$


where $A C[0, T]$ denotes the space of absolutely continuous functions on $[0, T]$. So, $\Lambda_{r, T}$ can be viewed as a tube of radius $r$ around the trajectory of the Wiener process on the interval $[0, T]$. Note further that $\Lambda_{r, T}$ falls into the category of sets of Theorem 2.2.5, i.e. $\Lambda_{r, T}$ is an invariant $K$-minimal set with respect to $\left(L^{1}[0, T], L^{\infty}[0, T]\right)$.

Now, in Paper IV it is proved that there exists a constant $\mathcal{C} \in(0, \infty)$ such that for any fixed $r>0$

$$
\inf _{f \in \Lambda_{r, T}} r\left(\frac{1}{T} \int_{0}^{T} f^{\prime}(t)^{2} d t\right)^{1 / 2} \rightarrow \mathcal{C}
$$

both in $L_{q}, q>0$, and almost surely as $T \rightarrow \infty$. An interpretation of (2.6) can be given along these lines: an absolutely continuous function must asymptotically spend on average $\mathcal{C}^{2} / r^{2}$ amount of energy per unit of time if it is constrained to stay within the distance $r$ from the almost surely non-differentiable trajectory of $W$. The exact value of $\mathcal{C}$ is unknown at present. A lower bound of approximately 0.38 and an upper bound of $\frac{\pi}{2}$ of $\mathcal{C}$ is proved in Paper IV. We recall that the outcome of the simulations of the discrete problem was $\mathcal{C} \approx 0.63$ which in between the lower and upper bounds. 


\section{References}

[1] R. E. Barlow, D. J. Bartholomew, J. M. Bremner, and H. D. Brunk. Statistical Inference under Order Restrictions: The Theory and Application of Isotonic Regression. Wiley Series in Probability and Mathematical Statistics. John Wiley \& Sons, London, 1972.

[2] C. Bennett and R. Sharpley. Interpolation of Operators. Pure and Applied Mathematics, Vol. 129. Academic Press, Boston, MA, 1988.

[3] J. Bergh and J. Löfström. Interpolation Spaces. An Introduction. Grundlehren der mathematischen Wissenschaften, 223. Springer-Verlag, Berlin, 1976.

[4] Yu. A. Brudnyı̆ and N. Ya. Krugljak. Interpolation Functors and Interpolation Spaces, vol. 1. North-Holland Mathematical Library, Vol. 47. North-Holland, Amsterdam, 1991.

[5] A. P. Calderón. Intermediate spaces and interpolation. Studia Math. (Special series), 1:31-34, 1963.

[6] A. P. Calderón. Intermediate spaces and interpolation, the complex method. Studia Math., 24:113-190, 1964.

[7] A. P. Calderón. Spaces between $L^{1}$ and $L^{\infty}$ and the theorem of Marcinkiewicz. Studia Math., 26:273-299, 1966.

[8] G. B. Dantzig. A control problem of Bellman. Management Sci., 17:542-546, 1971.

[9] B. Grünbaum. Convex Polytopes. Graduate Texts in Mathematics, 221. Springer-Verlag, New York, second edition, 2003.

[10] G. H. Hardy, J. E. Littlewood, and G. Pólya. Some simple inequalities satisfied by convex functions. Messenger of Mathematics, 58:145-152, 1929.

[11] I. Karatzas and S. E. Shreve. Brownian Motion and Stochastic Calculus. Graduate Texts in Mathematics, 113. Springer-Verlag, New York, 1988.

[12] S. G. Kreŭn, Ju. I. Petunin, and E. M. Semenov. Interpolation of linear operators. Translations of Mathematical Monographs, Vol. 54. American Mathematical Society, Providence, R.I., 1982. 
[13] J. L. Lions. Une construction d'espaces d'interpolation. C. R. Acad. Sci. Paris, 251:1853-1855, 1960.

[14] J. L. Lions and J. Peetre. Sur une classe d'espaces d'interpolation. Inst. Hautes Études Sci. Publ. Math., 19:5-68, 1964.

[15] E. Mammen and S. van de Geer. Locally adaptive regression splines. Ann. Statist., 25:387-413, 1997.

[16] J. Marcinkiewicz. Sur l'interpolation d'opérations. C. R. Acad. Sci. Paris, 208:1272-1273, 1939.

[17] A. W. Marshall, I. Olkin, and B. C. Arnold. Inequalities: Theory of Majorization and Its Applications. Springer, New York, second edition, 2011.

[18] F. Modigliani and F. E. Hohn. Production planning over time and the nature of the expectation and planning horizon. Econometrica, 23:46-66, 1955.

[19] A. Muhammad, P. Johansson, and R. Forchheimer. Performance evaluation of rate-control strategies for real-time systems over a rate-varying channel. Journal of Communications, 5:390-399, 2010.

[20] J. Niyobuhungiro and E. Setterqvist. A new reiterative algorithm for the Rudin-Osher-Fatemi denoising model on the graph. In Proceedings of the 2nd International Conference on Intelligent Systems and Image Processing 2014, pages 81-88, September 2014. See http://liu.divaportal.org/smash/get/diva2:719719/FULLTEXT01.pdf for full version.

[21] M. Riesz. Sur les maxima des formes bilinéaires et sur les fonctionnelles linéaires. Acta Math., 49:465-497, 1927.

[22] J. D. Salehi, Z.-L. Zhang, J. Kurose, and D. Towsley. Supporting stored video: Reducing rate variability and end-to-end resource requirements through optimal smoothing. IEEE/ACM Trans. Networking, 6:397-410, 1998.

[23] O. Scherzer, M. Grasmair, H. Grossauer, M. Haltmeier, and F. Lenzen. Variational Methods in Imaging. Applied Mathematical Sciences, 167. Springer, New York, 2009.

[24] G. O. Thorin. An extension of a convexity theorem due to M. Riesz. Kungl. Fysiogr. Sällsk. i Lund Förh., 8:166-170, 1938. 


\section{Papers}

The articles associated with this thesis have been removed for copyright reasons. For more details about these see:

http://urn.kb.se/resolve?urn=urn:nbn:se:liu:diva-132421 